\title{
Simulación con un modelo hidrológico distribuido de tipo conceptual a escala diaria en una cuenca semiárida del río Lurín, Perú
}

\author{
Simulation with a conceptual distributed hydrological model on a \\ daily scale in a semi-arid basin of the Lurin River, Peru \\ Diego Meléndez Saldaña ${ }^{1}$; Lía Ramos-Fernández*2; \\ Teresa Velásquez Bejarano²; Lisette Altamirano Gutiérrez ${ }^{1}$
}

\section{RESUMEN}

El río Lurín se ubica en la parte costera del Perú, cuyo clima es árido, con poca precipitación y un mal manejo de sus fuentes de agua. Esto ha ocasionado un insuficiente crecimiento de la agricultura y ganadería, así como la falta de información climática e hidrométrica, lo cual ha generado dificultades para la gestión del recurso hídrico en la cuenca. Se aplicó el modelo TETIS para la modelación hidrológica distribuida de la cuenca del río Lurín, para lo cual se utilizó información de precipitación, evapotranspiración y caudal, los mapas geomorfológicos (MED, celdas acumuladas, dirección del flujo, pendiente, velocidad), los mapas de parámetros hidrológicos de almacenamiento estático (Hu) y conductividad hidráulica del suelo (Ks), y el mapa de conductividad hidráulica del acuífero (Kp), el mapa de cobertura vegetal y la información del índice de vegetación $(\lambda)$ por tipo de cobertura. En la estación hidrométrica de Antapucro y San Damián se realizó la calibración y validación diaria del modelo. Se obtuvieron los índices de Nash (NSE), error cuadrático medio normalizado (RSR) y error en volumen (Ev) de $(0,7551 ; 0,4949 ; 0,701 \%)$ para calibración y $(0,7611 ; 0,4888 ;-14,629 \%)$ para validación, respectivamente. Los parámetros con mayor sensibilidad en el modelo son el factor corrector de la evapotranspiración (FC2) y el factor de interpolación de lluvia ( $\beta$ ). Se simularon los caudales diarios de 1969 al 2019, y del balance hídrico anual se obtuvo una oferta de $121,36 \mathrm{hm}^{3}$ y una demanda de $111,59 \mathrm{hm}^{3}$ que corresponde principalmente al uso agrario. En el balance hídrico se observa un excedente de enero a mayo de $58,44 \mathrm{hm}^{3}$, el cual es descargado al mar debido a que no existe suficiente infraestructura de almacenamiento en la cuenca; $y$ en los meses de junio a diciembre se presenta un déficit de $48.67 \mathrm{hm}^{3}$ debido a la escasez de lluvia.

Palabras clave: TETIS, Lurín, calibración, validación, simulación.

\section{ABSTRACT}

The Lurin river is located in the coastal part of Peru. Although, in general the climate is arid, in some month of the year in possible to have some precipitation. This situation is the main cause of deficits in water resources management, inefficient growth of agriculture and livestock, lack of climatic and hydrometric information in the Basin. The TETIS Model which is a spatially distributed hydrological has been applied to modeling the Lurin river basin, for which information on precipitation, evapotranspiration and flow, geomorphological maps (MED, accumulated cells, flow direction, slope, speed), maps of hydrological parameters of static storage $(\mathrm{Hu})$ and hydraulic conductivity of the soil $(\mathrm{Ks})$ and the map of hydraulic conductivity of the aquifer (Kp), the map of vegetation cover and the information referred to the index of vegetation ( $\lambda)$ by type of cover. In the hydrometric station of Antapucro and San Damian, the daily calibration and validation of the model were carried out. Obtaining the Nash indices (NSE), normalized mean square error (RSR) and volume error (Ev) of (0.7551, 0.4949, 0.701\%) for calibration and $(0.7611,0.4888,-14.629 \%)$ for validation, respectively; the parameters with the highest sensitivity in the model being the evapotranspiration correction factor $(F C 2)$ and the rain interpolation factor $(\beta)$. The daily flows were simulated from 1969 to 2019, the annual water balance an offer of $121.36 \mathrm{hm}^{3}$ and demand of $111.59 \mathrm{hm}^{3}$ were obtained, which corresponds mainly to agricultural use. In the water balance there is a surplus from January to May of $58.44 \mathrm{hm}^{3}$, which is discharged to the sea due to the inexistent reservoir infrastructure in the basin. In the others months, June to December, there is a deficit of $48.67 \mathrm{hm}^{3}$ due to the lack of rain.

Keywords: TETIS, Lurin, calibration, validation, simulation.

\footnotetext{
1 Área Experimental de Riego, UNALM, Av. La Molina s/n La Molina, Lima, Perú.

2 Departamento de Recursos Hídricos, UNALM, Av. La Molina s/n La Molina, Lima, Perú.

* Autor por correspondencia: liarf@lamolina.edu.pe
}

Fecha de Recepción: 15 de Octubre, 2020.

Fecha de Aceptación: 26 de Diciembre, 2020. 


\section{Introducción}

El uso conjunto de las aguas superficiales y subterráneas se requiere esencialmente para un desarrollo sostenible de gestión de recursos hídricos en cuencas fluviales. El crecimiento demográfico sin precedentes durante los últimos años, particularmente debido a la extensa urbanización, condujo a la sobreexplotación de los recursos de aguas superficiales y subterráneas dentro de las cuencas fluviales (Loliyana et al., 2020). Los modelos hidrológicos permiten la simulación del ciclo hidrológico y con ello disponer de información en aquellas cuencas con escasos datos hidrométricos usando datos de precipitación, geomorfología, fisiografía, cobertura vegetal, entre otros, para ayudar a responder preguntas relacionadas con la disponibilidad y gestión del recurso, junto con otras aplicaciones (Tseganeh et al., 2020). Los modelos hidrológicos distribuidos permiten disponer de información más detallada a nivel de toda la red de la cuenca, pero requieren mayores datos.

El modelo hidrológico distribuido TETIS es un modelo basado en una conceptualización del tipo tanque y el agua se mueve hacia abajo siempre que no se exceda la capacidad de salida del tanque. TETIS divide el suelo en dos capas horizontales, y se compone de 20 parámetros y un factor de corrección utilizado para ajustar al máximo la transpiración. Además, el modelo ofrece la posibilidad de activar un submodelo dinámico de vegetación. La producción de escorrentía se realiza mediante un balance hídrico en cada celda, el cual se representa en un esquema de seis tanques de almacenamiento conceptuales, o siete si la celda pertenece a la red de cauces. El flujo entre tanques depende del agua almacenada en ellos, el esquema conceptual adoptado, el tipo de tanque y las características morfológicas e hidrológicas del suelo de la celda (Francés et al., 2014; Puertes et al., 2019).

La cuenca del río Lurín en la parte alta se caracteriza por ser lluviosa principalmente en los meses de enero, febrero y marzo, con una temperatura semifrígida y húmeda. En la parte media la temperatura varía de templada a semifría, con un clima semiseco y sin lluvia en la mayoría de los meses; y en la parte baja tiene un clima semicálido y desértico, así como escasez de lluvia en todas las estaciones del año y una humedad relativa clasificada como húmeda. Por ello se denomina cuenca semiárida.

Álvarez y Villaverde (2015) utilizaron el modelo STREAM a escala temporal mensual y escala espacial de $1 \mathrm{~km}$, para evaluar la disponibilidad hídrica actual y futura en la cuenca Lurín. Si bien los caudales promedios mensuales son aquellos que se usan en la mayoría de los análisis de disponibilidad hídrica, tal como explican Sudheer et al. (2007), la calibración de un modelo hidrológico debe ser realizada a nivel diario, a fin de preservar de manera más real el comportamiento hidrológico de la cuenca. Esto se reafirma con el estudio de Nourani et al. (2011), quienes en términos de modelación obtuvieron mejores resultados a escala diaria que a nivel mensual.

En este trabajo de investigación se formula y parametriza el modelo hidrológico distribuido TETIS con base física en el río Lurín a escala temporal diaria y escala espacial de $250 \mathrm{~m}$. Una vez calibrado y validado permitió simular información de caudales diarios en la red hidrográfica, a fin de determinar la oferta hídrica, los caudales máximos anuales para distintos periodos de retorno, el análisis de persistencia y el balance hídrico.

\section{Materiales y métodos}

Zona de estudio: el trabajo se realizó en la cuenca del río Lurín que desemboca en el océano Pacífico con un área drenada de $1,645.7 \mathrm{~km}^{2}$, un caudal medio de $4.3 \mathrm{~m}^{3} / \mathrm{s}$, una altitud máxima de $5242 \mathrm{msnm}$ y comprendida entre las coordenadas latitud Sur $11^{\circ} 80^{\prime}$ y $12^{\circ} 20^{\prime} \mathrm{S}$, longitud Oeste $76^{\circ}$ $10^{\prime}$ y $76^{\circ} 88^{\prime}$ (Figura 1).

El procedimiento secuencial seguido en el estudio se aprecia en la Figura 2.

Caracterización de la hidrometeorología: se recopilaron datos climáticos e hidrométricos que se obtuvieron de los registros de las estaciones de SENAMHI ubicadas en el interior y los alrededores de la cuenca Lurín. (Tabla 1). Se realizó el análisis exploratorio y la corrección de datos inconsistentes por el método del vector regional. La evapotranspiración de referencia (ETo) se calculó por el método de Hargreaves, previamente ajustada con el método de Penman-Monteith: ETo $=\mathrm{C} * \mathrm{Ra} *(\mathrm{Tm}+17,8) *\left(\mathrm{TD}^{\wedge} 0,5\right)$.

Siendo C, 0,00202 para la zona húmeda y 0,00242 para la zona seca, $\mathrm{Ra}$ es la radiación extraterrestre en $\mathrm{mm} / \mathrm{día}$, Tm es la temperatura 


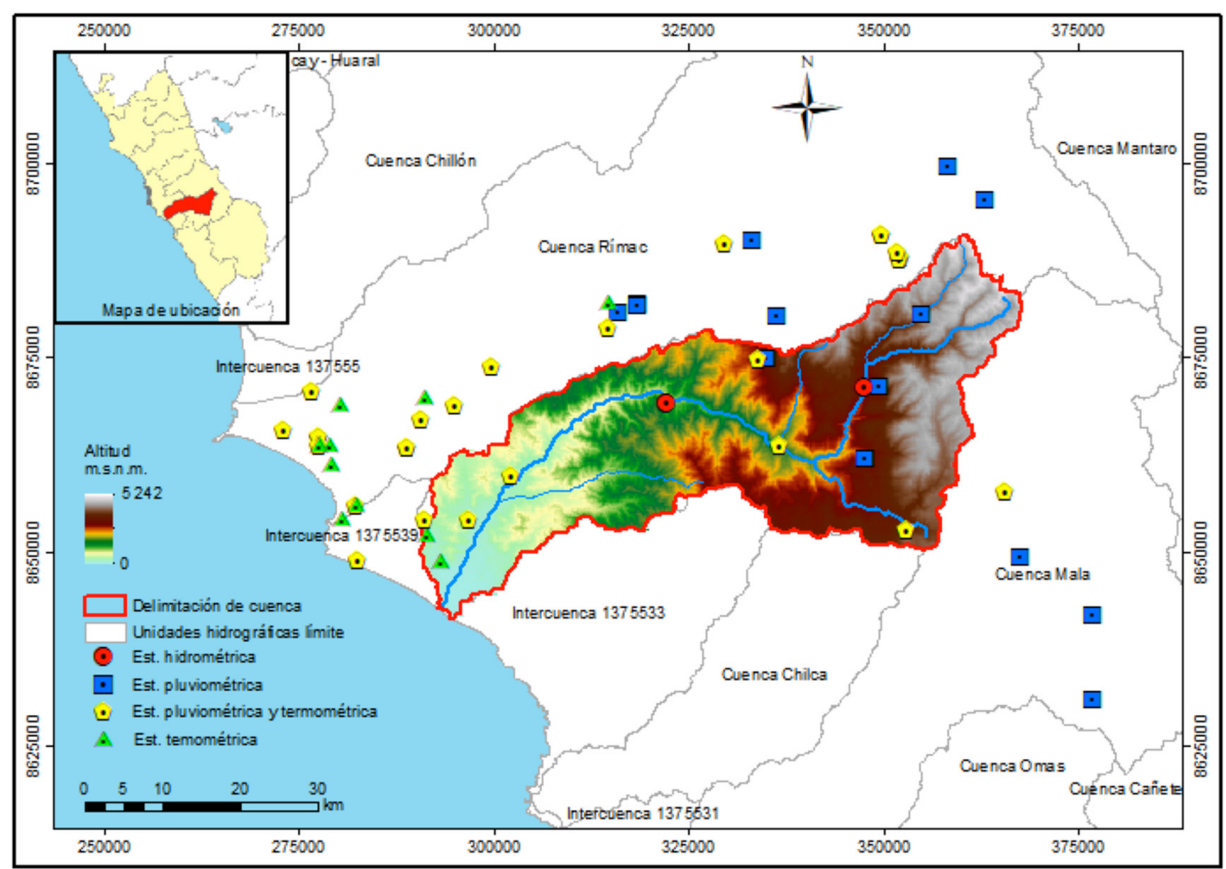

Figura 1. Variabilidad espacial de la altitud en la cuenca del río Lurín y ubicación de estaciones pluviométricas, termométricas e hidrométricas.

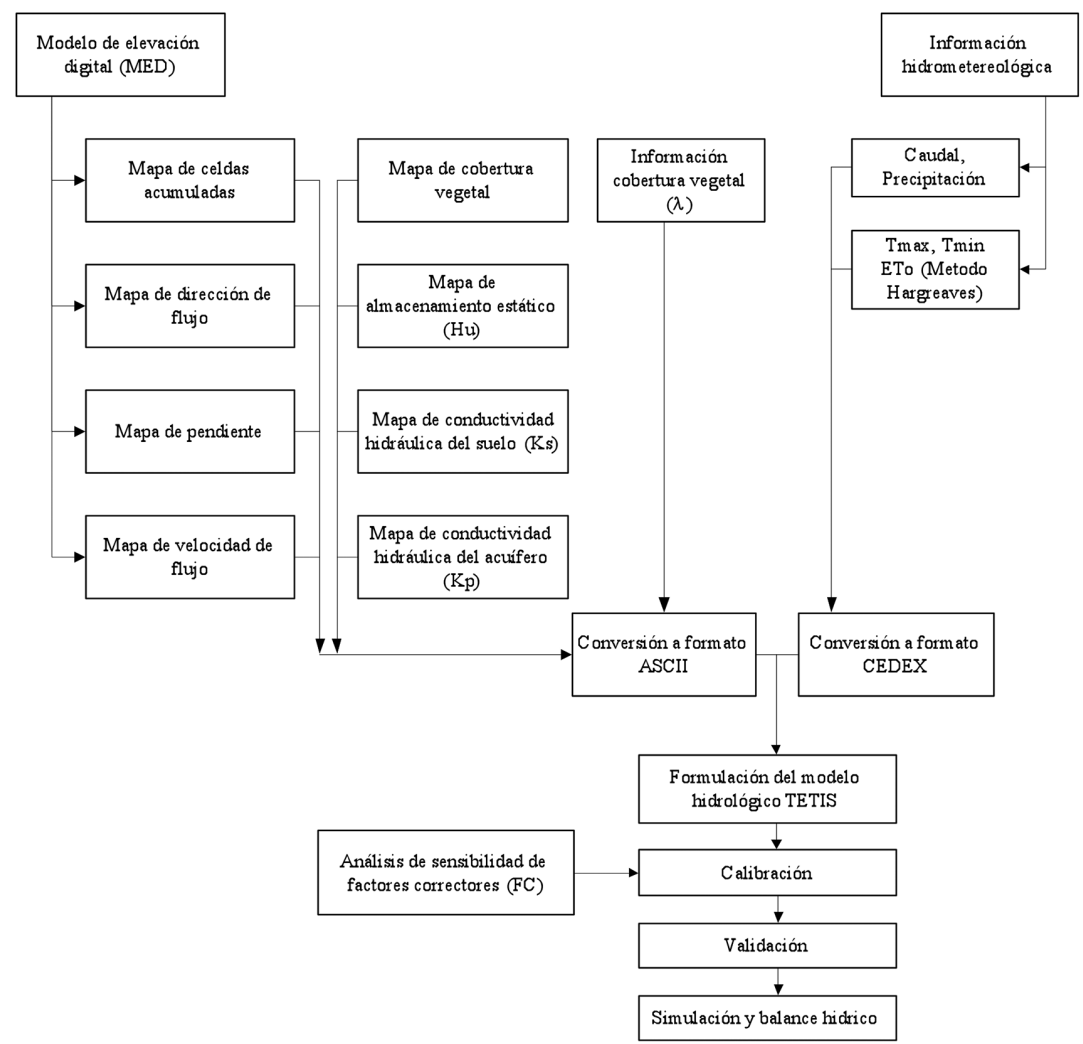

Figura 2. Procedimiento secuencial de la metodología que se siguió en el estudio. 
Tabla 1. Estaciones hidrometeorológicas de SENAMHI-Cuenca río Lurín.

\begin{tabular}{|c|c|c|c|c|c|c|}
\hline Estaciones & $\frac{\text { Altitud }}{(\mathrm{msnm})}$ & $\frac{\text { Longitud }}{\left({ }^{\circ} \mathrm{O}\right)}$ & $\frac{\text { Latitud }}{\left({ }^{\circ} \mathrm{S}\right)}$ & Datos* & Provincia & Distrito \\
\hline Antapucro & 1300 & 76,63 & 12,03 & $\mathrm{H}$ & Huarochiri & Antioquia \\
\hline San Damián & 3248 & 76,38 & 12,02 & $\mathrm{H}, \mathrm{P}$ & Huarochiri & San Damián \\
\hline Manchay & 229 & 76,83 & 12,14 & $\mathrm{H}$ & Lima & Cieneguilla \\
\hline Chalilla & 3975 & 76,33 & 11,93 & $\mathrm{P}$ & Huarochiri & San Damián \\
\hline Langa & 2860 & 76,42 & 12,13 & $\mathrm{P}$ & Huarochiri & Langa \\
\hline Santiago de Tuna & 2921 & 76,52 & 11,98 & $\mathrm{P}$ & Huarochiri & Santiago De Tuna \\
\hline Antioquia & 1839 & 76,50 & 12,08 & $P+T$ & Huarochiri & Antioquia \\
\hline Cieneguilla & 307 & 76,82 & 12,12 & $\mathrm{P}+\mathrm{T}$ & Lima & Cieneguilla \\
\hline Manchay Bajo & 164 & 76,87 & 12,17 & $\mathrm{P}+\mathrm{T}$ & Lima & Pachacamac \\
\hline San Lázaro de Escomarca & 3600 & 76,35 & 12,18 & $\mathrm{P}+\mathrm{T}$ & Huarochiri & Langa \\
\hline Santiago de Tuna & 2908 & 76,52 & 11,98 & $\mathrm{P}+\mathrm{T}$ & Huarochiri & Santiago De Tuna \\
\hline
\end{tabular}

* P: pluviométrica, T: termométrica, $\mathrm{P}+\mathrm{T}$ : pluviométrica y termométrica, H: hidrométrica Fuente: Elaborado con información del SENAMHI.

media diaria en ${ }^{\circ} \mathrm{C}$ y TD es la diferencia entre la temperatura máxima y la mínima diaria en ${ }^{\circ} \mathrm{C}$.

Con la información diaria de precipitación, evapotranspiración y caudal desde el 1/9/1969 al 31/12/2019, se preparó un archivo llamado episodio en formato CEDEX, requerido por el modelo hidrológico.

Caracterización de la geomorfología: a partir del modelo de elevación digital $(30$ x 30 m) se obtuvieron el mapa de celdas drenantes acumuladas, el mapa de dirección de flujo, el mapa de pendiente y el mapa de velocidad de flujo (Figura 3), que se adecuaron al formato ASCII requerido por el modelo TETIS.

Caracterización de la cobertura vegetal y parámetros hidrológicos: el mapa de cobertura vegetal se obtuvo del MINAM (2015), con once coberturas, predominando el desierto costero y el pajonal andino. Los factores de vegetación $(\lambda)$ por cada tipo de cobertura se muestran en las Figuras 4a y 4b. Los tipos de cobertura glaciar, cuerpos de agua, desierto costero y áreas urbanas tienen el valor de uno $(\lambda)$ por no existir vegetación. La textura, profundidad y capacidad de almacenamiento del suelo se obtuvieron de la base de datos Harmonized World Soil Database, a partir de lo cual se estimó el mapa de almacenamiento estático (Hu). Los mapas de conductividad hidráulica del suelo (Ks) y del acuífero (Kp) se estimaron a partir de información de Gleeson et al. (2014) y Gleeson et al. (2011), respectivamente, y la relación entre litología y conductividad hidráulica se obtuvo de Fetter (1994). Los parámetros hidrológicos variaron en un rango de $[9,1-106,0 \mathrm{~mm}],[0,02$ - $1,8 \mathrm{~mm} / \mathrm{h}]$ y $[0,001-0,99 \mathrm{~mm} / \mathrm{h}]$ para $\mathrm{Hu}, \mathrm{Ks}$ y Kp, respectivamente (Figuras 4 c, d, e). La información espacial se adecuó al formato ASCII requerido por el modelo TETIS.

Calibración y validación del modelo hidrológico: se empleó el modelo hidrológico distribuido TETIS a escala temporal diaria y escala espacial de $250 \mathrm{~m}$, y se ingresó al modelo la información de precipitación, evapotranspiración y caudal (archivo episodio), los mapas geomorfológicos (MED, celdas acumuladas, dirección del flujo, pendiente, velocidad), los mapas de parámetros hidrológicos( $\mathrm{Hu}, \mathrm{Ks}, \mathrm{Kp})$, el mapa de cobertura vegetal y la información del índice de vegetación ( $\lambda$ ) por tipo de cobertura. El modelo tiene nueve parámetros que se calibraron a través de factores correctores (FC) relacionados con el proceso de formación y distribución de la escorrentía. Previamente se realizó el análisis de sensibilidad de parámetros, y posteriormente la calibración manual y automática por el método de SCE-UA (Francés et al., 2014). La calibración se hizo en la estación Antapucro (1300 msnm) y la validación espacial en la estación San Damián (3248 mnsm). (Figura 1).

Se consideraron como índices de eficiencia de la modelación el índice de Nash- Stuled (NSE), error cuadrático medio normalizado (la razón entre la raíz de la desviación cuadrática media y desviación estándar de las observaciones) (RSR) y el error en volumen (Ev). 


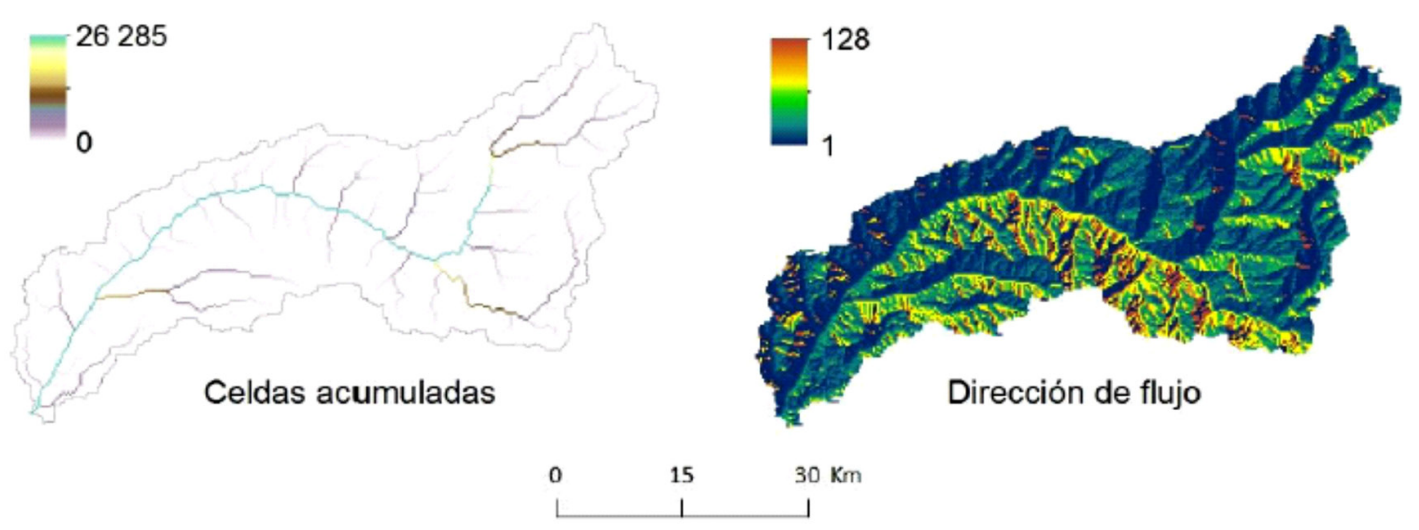

(a)

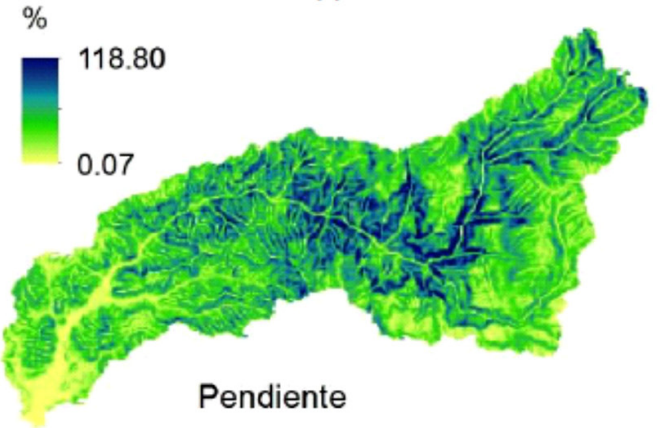

(b)

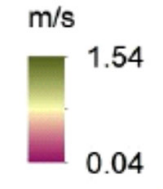

(c)

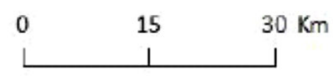

$30 \mathrm{Km}$

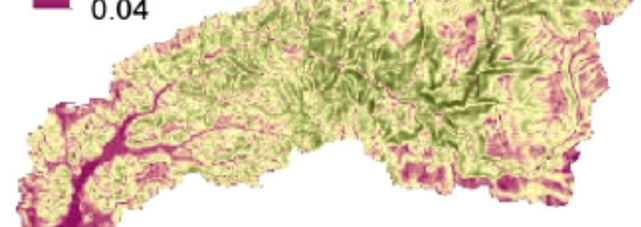

Velocidad de flujo

Figura 3. Variabilidad espacial de celdas drenantes acumuladas (a), dirección de flujo (b), pendiente (c) y velocidad de flujo (d).

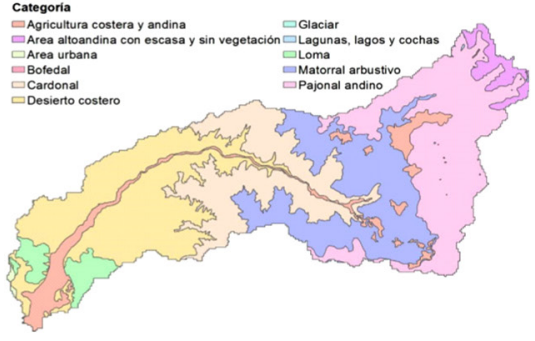

(a)

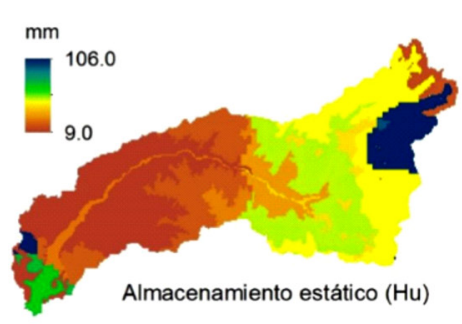

(c)

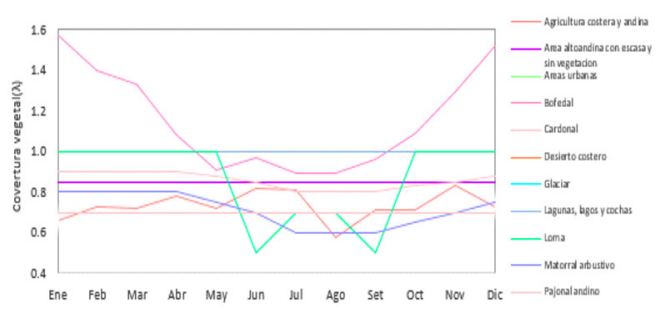

(b)

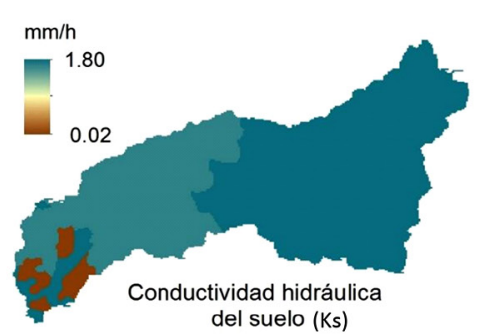

(d)

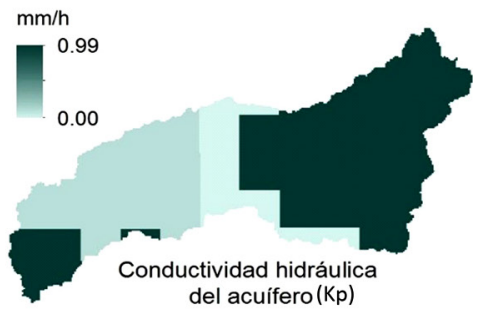

(e)

Figura 4. Variabilidad espacial del mapa de cobertura vegetal (a), diagrama mensual de cobertura vegetal $\lambda$ (b), mapa de almacenamiento estático $\mathrm{Hu}(\mathrm{c})$, conductividad hidráulica del suelo Ks (d), conductividad hidráulica del acuífero Kp (e). 
Estimación de la oferta hídrica a través de la simulación de caudales: con el modelo hidrológico previamente calibrado y validado se simuló el caudal diario del 1/9/1969 al 31/12/2019 en las subcuencas de San Damián y Antapucro; y se estimó la disponibilidad hídrica a los percentiles 50,75 y $95 \%$. En las márgenes del río Lurín existen 12 captaciones utilizadas por organizaciones de usuarios de riego (demanda agraria), así como tres captaciones para demanda poblacional en los distritos de Lurín, Pachacamac y Cieneguilla, entre otras demandas. Esta información se empleó en el balance hídrico para obtener el superávit o déficit hídrico mensual en la cuenca.

\section{Resultados y discusión}

Análisis de sensibilidad de parámetros: de los nueve parámetros del modelo, los que tienen mayor influencia son el factor corrector de la evapotranspiración (FC2) y el factor de interpolación de lluvia $(\beta)$, los cuales causan efectos opuestos, ya que el factor $\beta$ incrementa el agua proveniente de las lluvias, lo cual aumenta el caudal, mientras que el FC2 al aumentar, disminuye el agua disponible para la generación de caudal y viceversa. En la Figura 5 se muestra la mayor pendiente de la recta en FC2 y $\beta$.

Calibración y validación: en la Figura $6 \mathrm{a}$ se presenta el hidrograma diario de los caudales observados y simulados de la calibración en Antapucro y en la Figura 6b la validación en San
Damián. En la Tabla 2 se muestran los factores correctores obtenidos de la calibración, así como los índices de eficiencia de la calibración $(0,7535$, $0,4965,6,106 \%)$ y validación $(0,7611,0,4888$, $-14,629 \%)$ según índice de Nash (NSE), error cuadrático medio normalizado (RSR) y error en volumen (Ev), respectivamente. Estos índices, de acuerdo con Moriasi et al. (2007), muestran una calificación de "muy bueno" y "bueno" para calibración y validación, respectivamente.

Simulación de caudales diarios: se simularon los caudales diarios de 1969 al 2019 en las estaciones de San Damián y Antapucro. En las Figuras 7a y 7b se presenta la oferta hídrica para una probabilidad de ocurrencia del 50, 75 y $95 \%$. La información de estas estaciones se completó con el modelo hidrológico.

Balance hídrico: en la Figura 8 se muestran las demandas ubicadas aguas abajo del punto de control del balance hídrico, separadas de acuerdo a su uso, considerando como flujo de entrada la oferta en Antapucro a un $75 \%$ de persistencia. Se obtiene una oferta hídrica anual de $123,62 \mathrm{hm}^{3}$ y una demanda total de $111,60 \mathrm{hm}^{3}$ que corresponde principalmente al uso agrario. En la Figura 9 se observa un excedente de enero a mayo de $59,30 \mathrm{hm}^{3}$, el cual es descargado al mar debido a que no existe suficiente infraestructura de almacenamiento en la cuenca; y en los meses de junio a diciembre se presenta un déficit de $47,28 \mathrm{hm}^{3}$ debido a la escasez de lluvia.
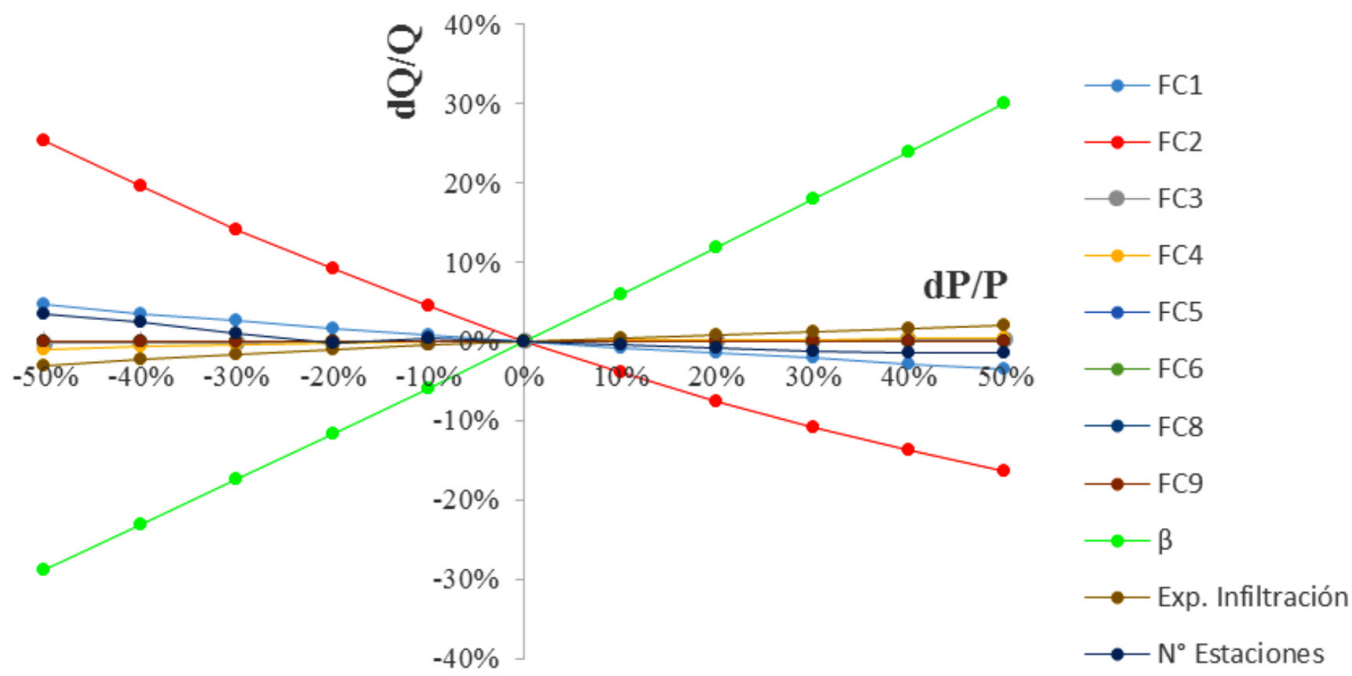

Figura 5. Variación porcentual del caudal promedio respecto a la variación de los factores correctores de los parámetros del modelo en la calibración. 

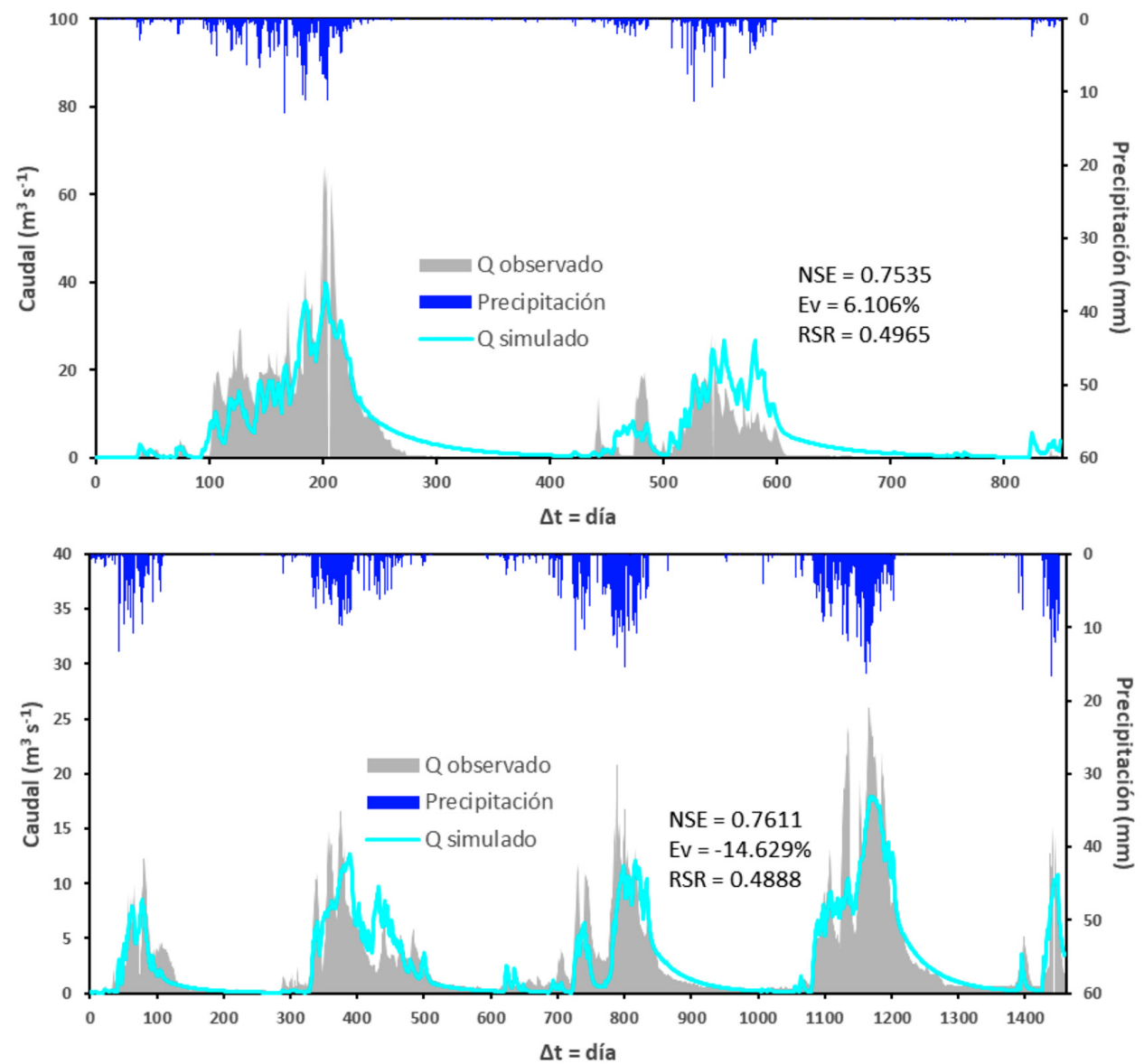

Figura 6. Hidrograma diario del caudal observado y simulado de la calibración en Antapucro, periodo de análisis del 1/9/1981 al 29/2/1984 (a, panel superior) y validación en San Damián, periodo de análisis del 1/9/1974 al 31/8/1977 (b, panel inferior).

Tabla 2. Factores correctores e índices de eficiencia de la calibración y validación del modelo TETIS.

\begin{tabular}{lcr}
\hline \multicolumn{1}{c}{ Factores correctores } & Símbolo & \multicolumn{1}{c}{ Valor } \\
\hline Almacenamiento estático & FC-1 & 0,941900 \\
Evapotranspiración & FC-2 & 0,999300 \\
Infiltración & FC-3 & 0,052400 \\
Escorrentía directa & FC-4 & 0,000080 \\
Percolación & FC-5 & 0,00150 \\
Interflujo & FC-6 & 2503,07129 \\
Pérdidas subterráneas & FC-7 & 0,00000 \\
Flujo base & FC-8 & 434,43051 \\
Velocidad en los cauces & FC-9 & 1,00000 \\
Factor de interpolación de lluvia & $\beta$ & 0,0002 \\
Exponente de infiltración al tanque estático & p1 & 2,90000 \\
\hline \multicolumn{1}{c}{ Índices de eficiencia } & Calibración & Validación \\
\hline NSE & 0,7535 & 0,7611 \\
RSR & 0,4965 & 0,4888 \\
Error en volumen $(\%)$ & 6,106 & $-14,629$ \\
Calificación & muy bueno & bueno \\
\hline
\end{tabular}



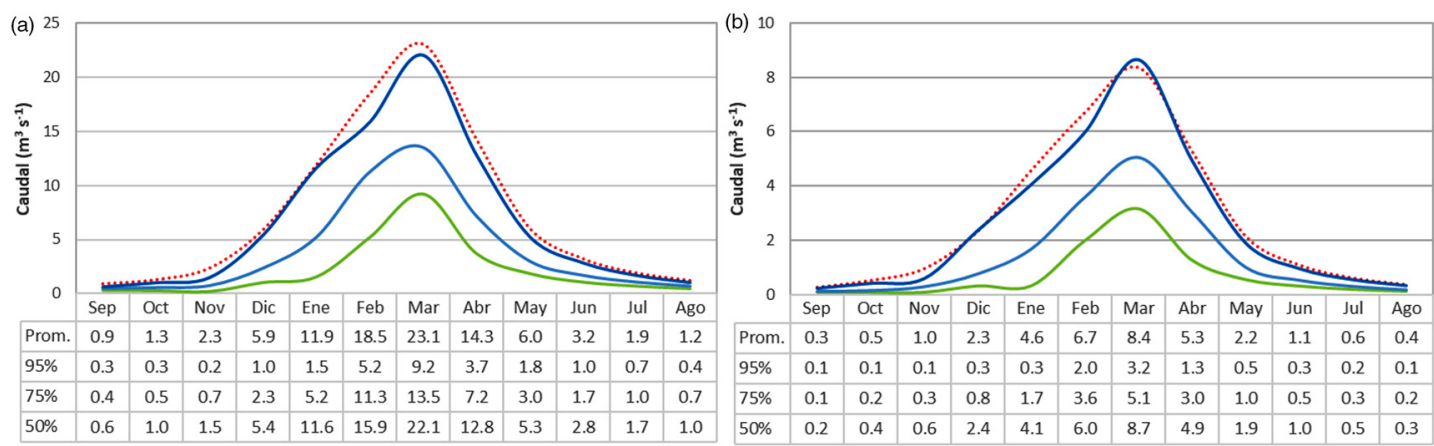

Figura 7. Caudal medio mensual de la oferta hídrica en Antapucro (a) y San Damián (b) para periodo de 1969 al 2019.

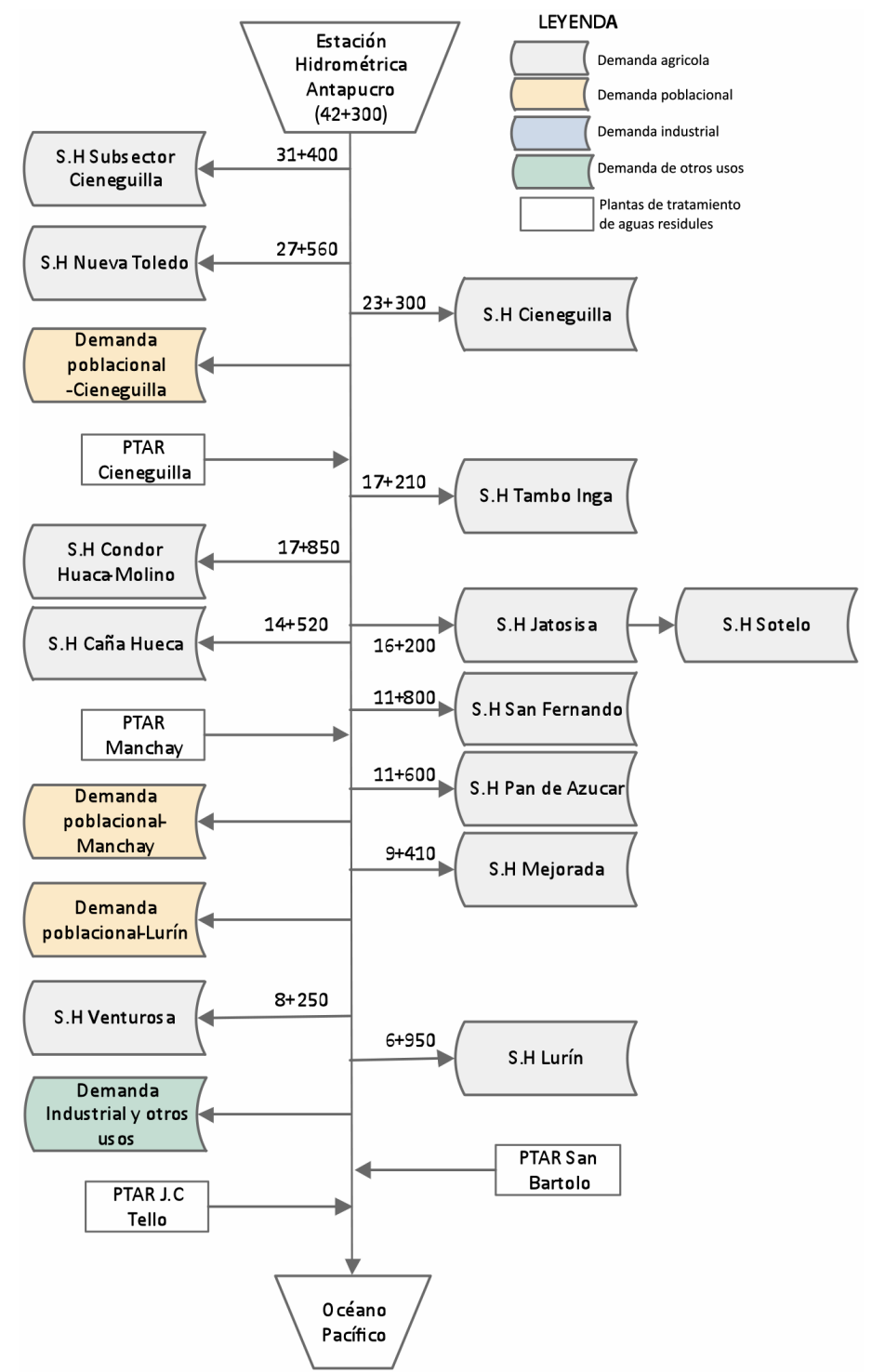

Figura 8. Puntos de oferta y demanda de agua en la cuenca Lurín a partir de la estación hidrométrica Antapucro. 


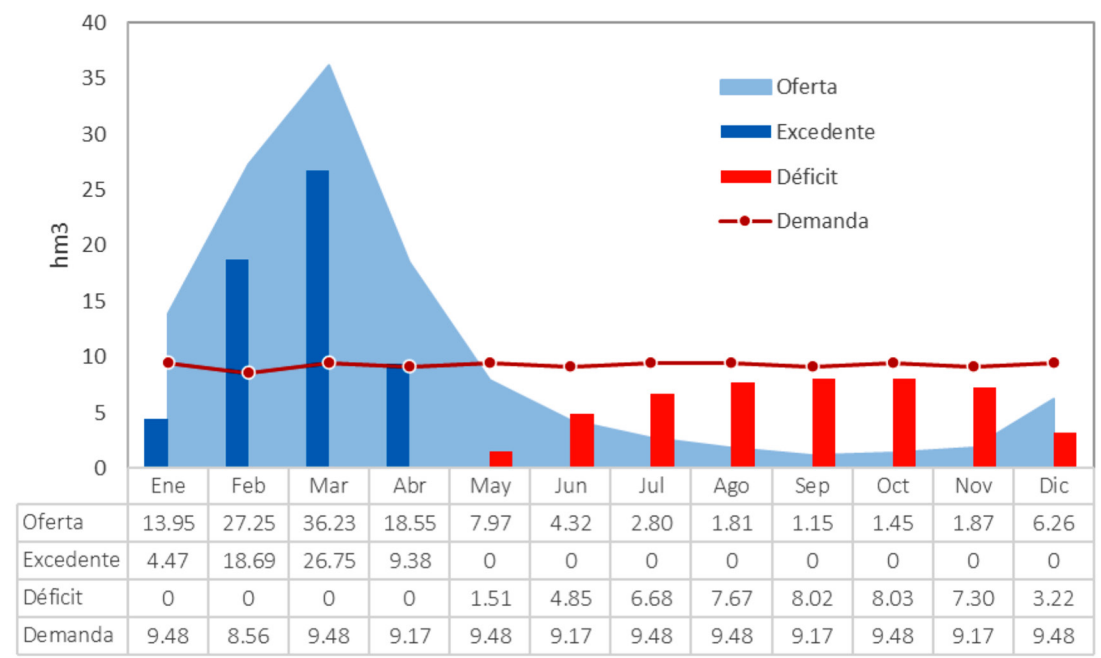

Figura 9. Balance hídrico en la cuenca del río Lurín aguas abajo de Antapucro (hm³).

\section{Conclusiones}

La calibración en Antapucro y la validación en San Damián mostraron resultados con el calificativo de "muy bueno" y "bueno" según el índice de Nash (NSE), el error cuadrático medio normalizado (RSR) y el error en volumen (Ev) con valores de $(0,7535$, $0,4965 ; 6,106 \%)$ para calibración y $(0,7611 ; 0,4888$; $-14,629 \%$ ) para validación, respectivamente. Los parámetros con mayor sensibilidad en el modelo son el factor corrector de la evapotranspiración (FC2) y el factor de interpolación de lluvia $(\beta)$.

Del balance hídrico anual se obtiene una oferta de $123,62 \mathrm{hm}^{3}$ y una demanda de $111,60 \mathrm{hm}^{3}$ que corresponde principalmente al uso agrario. En el balance hídrico se observa un excedente de enero a mayo de $59,30 \mathrm{hm}^{3}$, el cual es descargado al mar debido a que no existe suficiente infraestructura de almacenamiento en la cuenca; y en los meses de junio a diciembre se presenta un déficit de $47,28 \mathrm{hm}^{3}$ debido a la escasez de lluvia.

\section{Agradecimiento}

Este trabajo fue financiado por el CONCYTECFONDECYT en el marco de la convocatoria Proyecto Investigación Básica y Aplicada, 2017-02 [Convenio No 157-2017-FONDECYT]

\section{Literatura Citada}

Álvarez, T.; Hubert, Villaverde; R.A.

2015. Balance hídrico futuro en la cuenca del río Lurín a través de la modelación hidrológica ante el cambio climático. Lima, Perú, Universidad Nacional Agraria La Molina. pp. 2-3.

Autoridad Nacional del Agua, Perú -Observatorio del Agua Chillón - Cooperación Alemana.

2019. Diagnóstico Inicial para el Plan de Gestión de Recursos Hídricos de las cuencas Chillón, Rímac, Lurín y Chilca. Lima, Perú. 151 p.

Fetter, C.W.

1994. Applied Hydrogeology. 4 ed. Upper Saddle River, Estados Unidos de América, Prentice-Hall. 598 p.

Francés, F.; Vélez, J.

2014. Descripción del Modelo Conceptual Distribuido de Simulación Hidrológica TETIS v.8. Valencia, España, UPV. $86 \mathrm{p}$.
Gleeson, T.; Moosdorf, N.; Hartmann, J.; van Beek, L.P.H. 2014. A glimpse beneath earth's surface: GLobal HYdrogeology MaPS (GLHYMPS) of permeability and porosity. Medford, Massachusetts, Estados Unidos de América, CUAHSI. Gleeson, T.; Smith, L.; Moosdorf, N.; Hartmann, J.; Dürr, H.H.; Manning, A.H.; van Beek, L.P.H.; Jellinek, A.M.

2011. Global distribution of permeability. s. 1., Wiley.

Loliyana, V.D.; Patel, P.L.

2020. A physics based distributed integrated hydrological model in prediction of water balance of a semi-arid catchment in India. Journal Pre-proof Environmental Modelling and Software, 1: 1-43.

MINAM.

2015. Mapa Nacional de Cobertura Vegetal. Ministerio del Ambiente. Lima, Perú, MINAM.

Moriasi, D.N.; Arnold, J.G.; Van Liew, M.W.; Bingner, R.L.; Harmel, R.D.; Veith, T.L. 
2007. Model Evaluation Guidelines for Systematic Quantification of Accuracy in Watershed Simulations. American Society of Agricultural and Biological Engineers, 50(3): 885-900. Nourani, V.; Roughani, A.M.; Gebremichael, M.

2011. Top model for capability for rainfall-runoff modeling of the ammameh watershed at different time scales using different terrain algorithms. Journal of Urban and Environmental Engineering, 5(1): 1-14.

Puertes, C.; Lidón, A.; Echeverría, C.; Bautista, I.; GonzálezSanchis, M.; D. del Campo, A.; Francés, F.

2019. Explaining the hydrological behaviour of facultative phreatophytes using a multi-variable and multi- objective modelling approach, Journal of Hydrology, 575: 395-407.

Sudheer, K.P.; Chaubey, I.; Garg, V.; Migliaccio, K.W.

2007. Impact of time-scale of the calibration objective function on the performance of watershed models. Florida, Estados Unidos de América. Revista Hydrological Processes. 21(25): 3409-3419.

Tseganeh Z.G.; Nazmus S.S.; David G.T.; Pabitra D.

2020. HydroDS: Data Services in Support of Physically Based, Distributed Hydrological Models, Journal Preproof Environmental Modelling and Software, 1:1-42. 